\title{
PROSPECTIVE STUDY ON COMPARISON OF INTRAOPERATIVE FROZEN SECTION AND CYTOLOGICAL TECHNIQUES AS DIAGNOSTIC MODALITIES OF OVARIAN MASS LESIONS
}

\author{
Sonal Dwivedi', Riddhi Jaiswal2, Nisha Singh ${ }^{3}$, Ajay Kr Singh ${ }^{4}$, Madhumati Goel ${ }^{5}$ \\ 1 Junior Resident, Department of Pathology, KGMU, Lucknow. \\ ${ }_{2}^{2}$ Associate Professor, Department of Pathology, KGMU, Lucknow. \\ 3 Professor, Department of Obstetrics and Gynaecology, KGMU, Lucknow. \\ ${ }^{4}$ Associate Professor, Department of Pathology, KGMU, Lucknow. \\ 5Professor, Department of Pathology, KGMU, Lucknow.
}

\section{ABSTRACT}

\section{BACKGROUND}

Frozen section and cytology in gynaecological pathology have received less attention till date. This research focusses on indications and limitations of per-operative diagnostic techniques in pathology. Ovarian neoplasms are heterogeneous group of benign and malignant tumours classified on different cells of origin. Histopathology, however, remains the gold standard in tissue diagnosis.

\section{MATERIALS AND METHODS}

A descriptive study was conducted including 31 cases. Frozen section was performed on tissue specimens received in normal saline on thermo scientific HM525 cryostat. Simultaneous scrape and imprint smears were prepared and all slides stained with H and E, Giemsa and read by two independent pathologists. Results were then compared with histopathology of each case.

\section{RESULTS}

Frozen section in ovarian lesions showed sensitivity, specificity, positive predictive value, negative predictive value and accuracy values of $85.7 \%, 100 \%, 100 \%, 87.5 \%$ and $92.9 \%$ respectively. Aspirate cytology showed sensitivity, specificity, positive predictive value, negative predictive value and accuracy values of $40 \%, 100 \%, 100 \%, 62.5 \%$ and $70 \%$ respectively. Imprint cytology showed sensitivity, specificity, positive predictive value, negative predictive value and accuracy values of $33.3 \%, 100 \%, 100 \%, 60 \%$ and $66.7 \%$.

\section{CONCLUSION}

Comparison of intraoperative histology and cytology show that though cytology provides supporting information preoperatively, frozen section examination is more reliable in deciding extent of surgery in ovarian mass lesions.

\section{KEYWORDS}

Frozen Section, Cytology, Ovarian Mass.

HOW TO CITE THIS ARTICLE: Dwivedi S, Jaiswal R, Singh N, et al. Prospective study on comparison of intraoperative frozen section and cytological techniques as diagnostic modalities of ovarian mass lesions. J. Evolution Med. Dent. Sci. 2017;6 (42): 33473354, DOI: $10.14260 / \mathrm{Jemds} / 2017 / 725$

\section{BACKGROUND}

Frozen section is of great use in surgical oncology; however, in ovarian pathology large as well as small masses can be of dubious nature. Hence, the part played by per-operative diagnosis gains importance. The following study focuses on adding to the literature, use of frozen section and intraoperative cytology in diagnosis of ovarian diseases. An intraoperative consultation in gynaecological pathology is indicated- (a) To ensure that the tissue sampled is adequate for diagnosis; (b) To determine the nature of a disease process; (c) To plan for appropriate ancillary studies; (d) To determine tumour spread; and (e) To assess the margins.[1,2,3]

Ovarian neoplasms are a heterogeneous group of benign and malignant tumours of epithelial, stromal and germ cell origin. Histopathology remains the ultimate gold standard in tissue diagnosis.[4,5]

Financial or Other, Competing Interest: None.

Submission 12-04-2017, Peer Review 13-05-2017,

Acceptance 19-05-2017, Published 25-05-2017.

Corresponding Author:

Dr. Riddhi Jaiswal,

Associate Professor

Department of Pathology,

KGMU, Lucknow, UP.

E-mail: riddhiadvay@gmail.com

DOI: $10.14260 /$ jemds $/ 2017 / 725$

\section{(c) (1) $(9)$}

Rapid diagnosis of surgically removed specimens has created many controversies and a single completely reliable method has not yet been developed. Frozen section is the most frequently used technique along with some centres utilising cytological techniques including imprint and scrape cytology and intra-operative Fine-Needle Aspiration Cytology (FNAC).[3,6,7] Few studies in experienced hands have shown its diagnostic efficacy to be almost $92 \%$. It is simple and cheap, not requiring special training. ${ }^{[8-10]}$ The disadvantages are few and experience yields perfection. It however neither distinguishes in situ from invading carcinoma nor evaluates depth of invasion. Thus, apart from its diagnostic role, intraoperative cytology can become a learning tool as well.[8,11,12] Scrape smear cytology is a modified imprint cytology, in which cells are scraped from inner surface of specimen.[11-12] Along with frozen section, it helps expand knowledge of cytology of ovarian lesions.[13,14,15] Intraoperative opinion resorts staging and modification of surgical procedure.

\section{MATERIALS AND METHODS Study Setting}

Conducted at the Department of Pathology, in co-ordination with Department of Obstetrics and Gynaecology, King George's Medical University. 


\section{Duration of Study}

1 Year (August 2015 - July 2016).

\section{Study Type}

Descriptive study.

\section{Study Group}

All patients with ovarian diseases that need surgical treatment.

\section{Sample Collection}

Cystectomy, Ovarian biopsy, Oophorectomy and Salpingooophorectomy, Omental and Peritoneal nodules in patients ranging from 12 - 70 years were included in study.

In this study, the accuracy of intraoperative cytology was assessed and the results are compared with frozen section and final histopathological diagnosis.

Each technique was evaluated independently, although the diagnosis transmitted to the surgeon was based upon the combination of the clinical, macroscopic, histological and cytological in formation. The results were compared with the final pathological diagnosis in each case and cases with discordant diagnosis were reviewed.

\section{Inclusion Criteria}

Ovarian diseases that need surgical treatment, both neoplastic and non-neoplastic.

\section{Exclusion Criteria}

1. Inadequate/Non-representative samples.

2. Patients refusing consent.

1) Frozen Section Technique

The gross specimen is received in normal saline in the laboratory.

1. Embedding Tissue- The selected piece of tissue is then placed on a metallic holder and must be oriented a certain way so that the future section will reveal proper spatial relationships. This orientation depends on the question being asked.

The tissue is embedded in soluble M1 cryomatrix medium and is then placed in the cryostat machine, where it is properly frozen.

2. Cryostat- Thermo Scientific Micro HM550 installed in department was used for frozen sectioning. Turnaround time for each frozen section was 10 - 15 minutes.

\section{Temperature}

The temperature should be at $-20^{\circ} \mathrm{C}$ for most tissues. For tissues with a large fat component, $-40^{\circ} \mathrm{C}$ is optimal. This temperature is critical for optimal sectioning. (If the temperature is too high, i.e. $-10^{\circ} \mathrm{C}$, the tissue will not stay frozen and firm and will not cut crisp.

If the temperature is too cold, i.e. $-50^{\circ} \mathrm{C}$, the tissue will crumble and become powder. The ideal tissue should cut like butter, smooth and in one piece).

3. Staining- Once the section is cut, the slides are stained using progressive haematoxylin and eosin staining.

4. Interpreting the Frozen Section- By two pathologists independently.

2) Aspirate Cytology Smear Technique

1. Aspirate from the ovarian mass is collected by applying negative pressure using a $10 \mathrm{~mL}$ syringe.

2. The aspirate is then centrifuged and the supernatant discarded.

3. A drop is poured on the slide and smeared.

4. Slide is fixed by putting into the alcohol.

5. Slide is then stained by Harris haematoxylin and eosin staining.

3) Imprint Cytology Smear Technique

1. Slide is taken and tissue is imprinted on it by pressing upon.

2. It is then fixed by putting into the alcohol.

3. Stained by Harris haematoxylin and eosin.

4) Haematoxylin and Eosin Staining Procedure

1. Dissolve all wax with xylene or by keeping it on hot plate.

2. After thorough dewaxing, slide is passed through several changes of progressively decreasing concentration alcohol to remove xylene and then thoroughly rinsed in water.

3. Slide is now stained with nuclear stain such as haematoxylin, which consists of a dye and a mordant.

4. Slide is dipped for 8 - 15 minutes in the stain and then rinsed in tap water.

5. Background staining is removed and also contrast is improved by dipping the slide in weak acid alcohol for 1 - 5 seconds.

6. After this thorough rinsing in tap water is required.

7. Slide is then dipped in counter stain, eosin.

8. Slide is then passed through increasing concentration of alcohol and then dried and dipped in xylene.

9. Xylene clears the tissue and renders slide completely transparent and then slide is mounted.

5) Giemsa Staining Procedure

1. Air dried smear prepared.

2. Giemsa stain is then poured over the smear and kept for 10 minutes.

3. After this a similar quantity of buffer is added over the smear covered with the Giemsa stain.

4. Washed in running water.

5. Air dried and the slides then mounted with the help of xylene and Canada balsam.

Our study was approved by the Ethical Committee, King George's Medical University, Lucknow, UP.

\section{Statistical Analysis Plan}

The statistical analysis was done using SPSS (Statistical Package for Social Sciences) Version 15.0 Statistical Analysis Software. The values were represented in number, percentage and mean \pm SD. Sensitivity, specificity, positive predictive value, negative predictive value and accuracy values were calculated for each of three modalities.

\section{RESULTS}

Age of patients ranged from 12 to 70 years. Majority of cases were aged between 31 and 50 years $(n=18 ; 58.1 \%)$. There were only $2(6.5 \%)$ cases in age group $21-30$ and $61-70$ years. Mean age of patients was $38.29 \pm 14.65$ years. 


\begin{tabular}{|c|c|c|}
\hline Specimen & No. of Cases & Percentage \\
\hline Ovarian mass & 18 & 58.1 \\
\hline Uterus with adnexa & 8 & 25.8 \\
\hline Others & 5 & 16.1 \\
\hline Ovarian biopsy & 1 & 3.2 \\
\hline Ovary with gall bladder & 1 & 3.2 \\
\hline Right ovary with fallopian tube & 1 & 3.2 \\
\hline $\begin{array}{c}\text { Sigmoid colon, rectum, left } \\
\text { ovary }\end{array}$ & 1 & 3.2 \\
\hline \multicolumn{2}{|c|}{1} & 3.2 \\
\hline Tabo-ovarian mass 1. Distribution of Cases according to Specimen Type \\
\hline
\end{tabular}

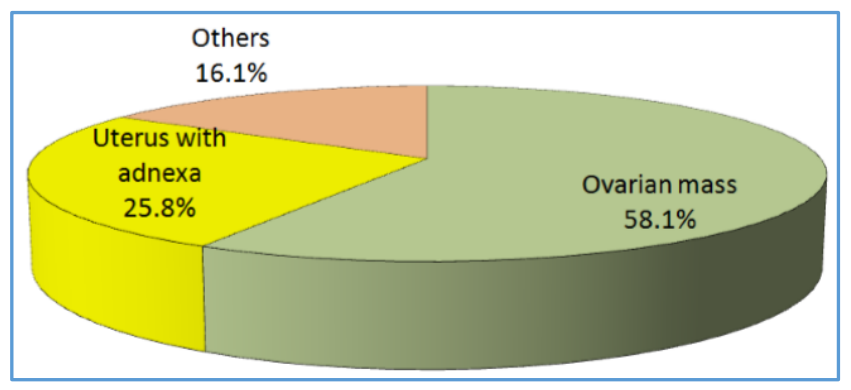

Graph 1

Majority of specimen were obtained from ovarian mass ( $n=18 ; 58.1 \%)$. A total of 8 specimens $(25.8 \%)$ were obtained from uterus with adnexa. Among remaining $5(16.1 \%)$ cases, one each (3.2\%) was obtained from ovarian biopsy, ovary with gall bladder, right fallopian tube, sigmoid colon-rectum with left ovary and tubo-ovarian mass respectively.

\begin{tabular}{|c|c|c|c|c|}
\hline Sl. No. & Aspirate & Frozen & Imprint & Histology \\
\hline 1. & Positive for malignant cells & Mixed germ cell tumour & Paucicellular & $\begin{array}{l}\text { Malignant mixed germ cell } \\
\text { tumour }\end{array}$ \\
\hline 2. & $\begin{array}{l}\text { Hemosiderin-laden } \\
\text { macrophages }\end{array}$ & Cyst & Hemosiderin-laden macrophages & Follicular cyst \\
\hline 3. & $\begin{array}{l}\text { Paucicellular comprising of } \\
\text { mesothelial cells, RBC's and } \\
\text { inflammatory cells }\end{array}$ & Cyst & Paucicellular & Simple cyst \\
\hline 4. & Acellular & Cyst & Paucicellular & Autolysed tissue only \\
\hline 5. & Mucoid material & Necrotic material & Suspicious atypical cell clusters & Mixed germ cell tumour \\
\hline 6. & Negative & Suspicious for malignancy & Degenerated cells & $\begin{array}{c}\text { Left ovary- Granulosa cell } \\
\text { tumour }\end{array}$ \\
\hline 7. & $\begin{array}{l}\text { Benign columnar epithelial } \\
\text { cells }\end{array}$ & Suspicious for malignancy & Negative & $\begin{array}{c}\text { Mucinous cyst } \\
\text { adenocarcinoma }\end{array}$ \\
\hline 8. & Proteinaceous material only & Cyst & Haemorrhage only & Mature teratoma \\
\hline 9. & Positive for malignant cells & $\begin{array}{l}\text { Papillary serous cyst } \\
\text { adenocarcinoma }\end{array}$ & Degenerated suspicious cells & High-grade serous carcinoma \\
\hline 10. & $\begin{array}{c}\text { Few squamous epithelial } \\
\text { cells }\end{array}$ & Cyst & $\begin{array}{l}\text { Pultaceous material, necrotic } \\
\text { material }\end{array}$ & Mature teratoma \\
\hline 11. & Negative & Suspicious for malignancy & Positive for suspicious cells & High-grade serous carcinoma \\
\hline 12. & Negative & $\begin{array}{c}\text { Papillary serous } \\
\text { cystadenocarcinoma }\end{array}$ & $\begin{array}{l}\text { Small cluster of epithelial cells, } \\
\text { mesothelial cells }\end{array}$ & $\begin{array}{c}\text { Papillary serous cyst } \\
\text { adenocarcinoma }\end{array}$ \\
\hline 13. & Negative & $\begin{array}{c}\text { Benign-to-borderline serous } \\
\text { cyst adenoma }\end{array}$ & $\begin{array}{c}\text { Mesothelial cells and bland } \\
\text { epithelial cells }\end{array}$ & $\begin{array}{c}\text { Borderline serous cyst } \\
\text { adenoma }\end{array}$ \\
\hline 14. & Negative & Fibrocollagenous tissue & Negative & Simple cyst \\
\hline 15. & $\begin{array}{l}\text { Spindled and inflammatory } \\
\text { cells }\end{array}$ & Endometriosis & Stromal and inflammatory cells & Endometriosis \\
\hline 16. & Anucleated squames & Teratoma & Anucleated squames & Mature teratoma \\
\hline 17. & Positive for tumour cells & Yolk sac tumour & Positive for tumour cells & $\begin{array}{l}\text { Malignant mixed germ cell } \\
\text { tumour }\end{array}$ \\
\hline 18. & Negative & $\begin{array}{c}\text { Granulosa cell tumour/Non- } \\
\text { Hodgkin's lymphoma }\end{array}$ & Positive & Granulosa cell tumour \\
\hline 19. & Negative & Benign serous cyst & Sheets of monolayered cells & Benign serous cyst \\
\hline 20. & Negative & Yolk sac tumour & Negative & Granulosa cell tumour \\
\hline 21. & Negative & Simple Cyst & Negative & Simple mucinous cyst \\
\hline 22. & Negative & Haemorrhagic cyst & Negative & Endometriosis \\
\hline 23. & Negative & No evidence of malignancy & Negative & No e/o malignancy \\
\hline 24. & $\begin{array}{l}\text { Hemosiderin-laden } \\
\text { macrophages }\end{array}$ & Endometriosis & Hemosiderin-laden macrophages & Endometriosis \\
\hline 25. & Positive for malignant cells & Serous cyst adenocarcinoma & Negative for malignant cells & $\begin{array}{c}\text { Papillary serous cyst } \\
\text { adenocarcinoma }\end{array}$ \\
\hline 26. & Paucicellular & Suspicious for malignancy & Negative for malignant cells & Malignant ovarian tumour \\
\hline 27. & Paucicellular & Benign cyst & Negative for malignant cells & Malignant ovarian tumour \\
\hline 28. & Not done & Germ cell tumour & Not done & Germ cell tumour \\
\hline 29. & Positive for tumour cells & Not done & Positive for malignant cells & Granulosa cell tumour \\
\hline 30. & Negative for tumour cells & Not done & Negative for malignant cells & Mature teratoma \\
\hline 31. & Positive for tumour cells & Not done & Positive for malignant cells & $\begin{array}{c}\text { Mucinous cyst } \\
\text { adenocarcinoma }\end{array}$ \\
\hline
\end{tabular}




\begin{tabular}{|c|c|c|}
\hline Specimen & No. of Cases & Percentage \\
\hline Benign & 16 & 57.1 \\
\hline Benign ovarian cyst & 10 & 35.71 \\
\hline Endometriosis & 2 & 7.1 \\
\hline Fibrocollagenous tissue & 1 & 3.6 \\
\hline Necrotic material & 1 & 3.6 \\
\hline No evidence of malignancy & 1 & 3.6 \\
\hline Teratoma & 1 & 3.6 \\
\hline Malignant & 12 & 42.9 \\
\hline Serous cyst adenocarcinoma & 3 & 10.7 \\
\hline Suspicious for malignancy & 4 & 14.3 \\
\hline Yolk sac tumour & 2 & 7.1 \\
\hline Germ cell tumour & 2 & 7.1 \\
\hline Granulosa cell tumour/NHL & 1 & 3.6 \\
\hline \multicolumn{3}{|c|}{ Table 3. Diagnoses Based on Frozen Section Evaluation } \\
\hline
\end{tabular}

Frozen section could be evaluated in 28/31 (90.3\%) cases, out of which 16 (57.1\%) were diagnosed as benign and 12 (42.9\%) as malignant.

Among cases diagnosed as benign, maximum $(n=10$; $35.7 \%$ ) were diagnosed as benign ovarian cyst, 2 cases (7.1\%) were diagnosed as endometriosis and one each (3.6\%) reported as fibrocollagenous tissue, necrotic material, teratoma and absence of malignancy.

Among cases diagnosed as malignant, maximum were diagnosed as suspicious for malignancy ( $n=4 ; 14.3 \%)$ followed by serous cyst adenocarcinoma (10.7\%), yolk sac tumour $(\mathrm{n}=2 ; 7.1 \%$ each) and germ cell tumour $(\mathrm{n}=2 ; 7.1 \%)$ and granulosa cell tumour/NHL $(\mathrm{n}=1 ; 3.6 \%)$.

\begin{tabular}{|c|c|c|}
\hline Specimen & No. of Cases & Percentage \\
\hline Benign & 24 & 80.0 \\
\hline Acellular & 1 & 3.3 \\
\hline Anucleated squames & 1 & 3.3 \\
\hline Benign columnar cells & 1 & 3.3 \\
\hline Few squamous epithelial cells & 1 & 3.3 \\
\hline Hemosiderin-laden macrophages & 2 & 6.7 \\
\hline Mucoid material & 1 & 3.3 \\
\hline Negative for Malignant Cells & 12 & 40.0 \\
\hline Paucicellular & 2 & 6.7 \\
\hline $\begin{array}{c}\text { Paucicellular comprising of } \\
\text { mesothelial cells, red blood cells } \\
\text { and inflammatory cells }\end{array}$ & 1 & 3.3 \\
\hline Proteinaceous material only & 1 & 3.3 \\
\hline Spindle and inflammatory cells & 1 & 3.3 \\
\hline Positive for Malignant Cells & 6 & 20.0 \\
\hline
\end{tabular}

Aspiration cytology could be performed in 30 cases. Out of these, 24 (80\%) were diagnosed as benign and remaining 6 (20\%) were diagnosed as positive for malignant cells.

Among cases diagnosed as benign, a total of 12 cases (40\%) were negative for malignant cells. In 2 (6.7\%) cases hemosiderin-laden macrophages were seen, while another 2 $(6.7 \%)$ were paucicellular. There was one $(3.3 \%)$ case each, which were reported as acellular, anucleated squames, presence of few squamous epithelial cells, mucoid material, paucicellular comprising of mesothelial cells, RBCs and inflammatory cells, proteinaceous material and spindle and inflammatory cells respectively.

\begin{tabular}{|c|c|c|}
\hline Specimen & No. of Cases & Percentage \\
\hline Benign & $\mathbf{2 5}$ & $\mathbf{8 3 . 3}$ \\
\hline Negative & 10 & 33.3 \\
\hline Anucleated squames & 1 & 3.3 \\
\hline
\end{tabular}

\begin{tabular}{|c|c|c|}
\hline Degenerated cells & 1 & 3.3 \\
\hline Degenerated suspicious cells & 1 & 3.3 \\
\hline Hemorrhage only & 1 & 3.3 \\
\hline Hemosiderin-laden macrophages & 2 & 6.7 \\
\hline $\begin{array}{c}\text { Mesothelial cells and bland } \\
\text { epithelial cells }\end{array}$ & 1 & 3.3 \\
\hline Paucicellular & 3 & 10.0 \\
\hline Negative for tumour cells & 1 & 3.3 \\
\hline $\begin{array}{c}\text { Pultaceous material, necrotic } \\
\text { material }\end{array}$ & 1 & 3.3 \\
\hline Sheets of monolayered sheets & 1 & 3.3 \\
\hline $\begin{array}{c}\text { Small cluster of epithelial cells, } \\
\text { mesothelial cells }\end{array}$ & 1 & 3.3 \\
\hline Stromal and inflammatory cells & 1 & 3.3 \\
\hline Positive for Malignant Cells & 5 & 16.7 \\
\hline Table 5. Imprint Cytology Diagnosis \\
\hline
\end{tabular}

Imprint cytology could be performed in 30 cases. Of these, $25(83.3 \%)$ were diagnosed as benign and remaining 5 (16.7\%) were diagnosed as malignant.

A total of 25 cases were diagnosed as benign, 10 (33.3\%) were negative, 3 cases were reported as (10\%) paucicellular, 2 cases (6.7\%) were reported as hemosiderin-laden macrophages, 1 case $(3.3 \%)$ each had anucleated squames, degenerated cells, degenerated suspicious cells, haemorrhage only, mesothelial cells with bland epithelial cells, negative for tumour cells, pultaceous/necrotic material, sheets of monolayered cells, small cluster of epithelial cells/mesothelial cells and stromal with inflammatory cells respectively.

\begin{tabular}{|c|c|c|}
\hline Specimen & No. of Cases & Percentage \\
\hline Benign & 15 & 48.4 \\
\hline Autolysed tissue only & 1 & 3.2 \\
\hline Benign serous cyst & 2 & 6.5 \\
\hline Endometriosis & 3 & 9.7 \\
\hline Follicular cyst & 1 & 3.2 \\
\hline Mature teratoma & 4 & 12.9 \\
\hline No evidence of malignancy & 1 & 3.2 \\
\hline Simple cyst & 2 & 6.5 \\
\hline Simple mucinous cyst & 1 & 3.2 \\
\hline Malignant & 16 & 51.6 \\
\hline Germ cell tumour & 4 & 12.9 \\
\hline Granulosa cell tumour & 4 & 12.9 \\
\hline Serous cyst adenocarcinoma & 4 & 12.9 \\
\hline $\begin{array}{c}\text { Mucinous cyst } \\
\text { adenocarcinoma }\end{array}$ & 2 & 6.5 \\
\hline Malignant ovarian tumour & 2 & 6.5 \\
\hline \multicolumn{3}{|c|}{ Table 6. Final Diagnosis $(n=31)$} \\
\hline
\end{tabular}

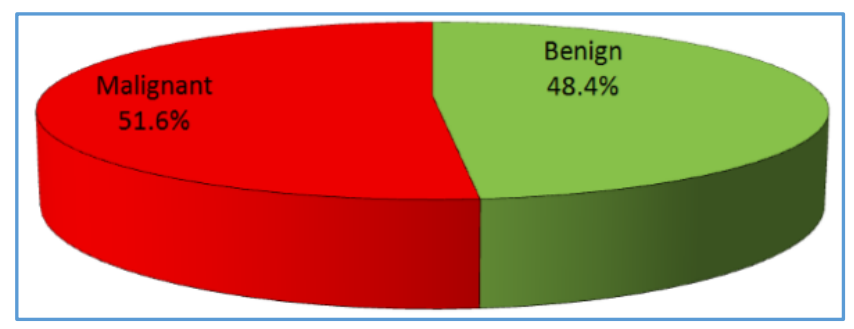

Graph 2 


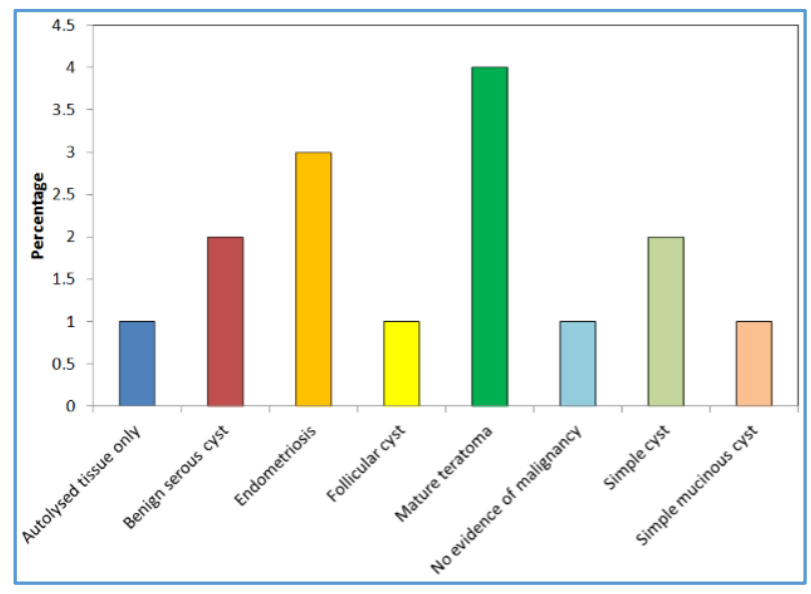

Graph 3

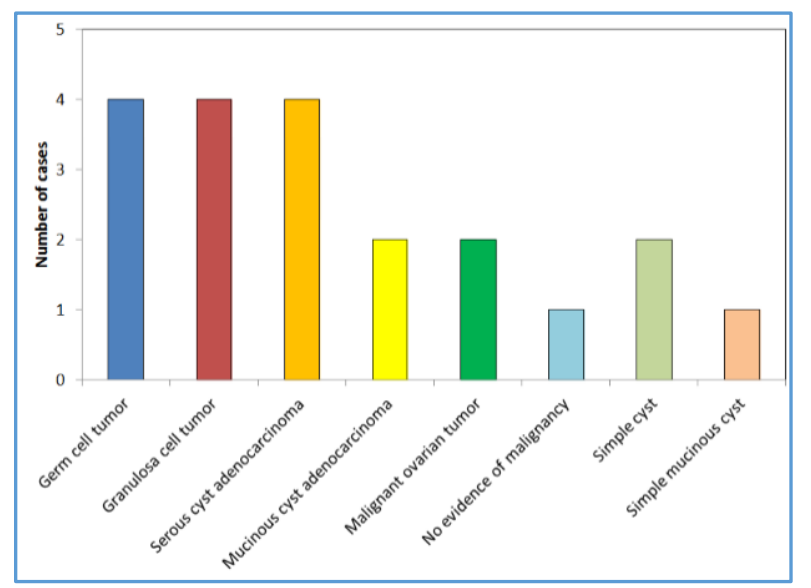

Graph 4

Final diagnosis based on histopathology was benign in 15 (48.4\%) and malignant in 16 (51.6\%) cases.

Among benign cases, the most common diagnosis was mature teratoma $(n=4 ; 12.9 \%)$ followed by endometriosis $(n=3 ; 9.7 \%)$, simple cyst and benign serous cyst in $2(6.5 \%)$ cases each. There was 01 case each reported as autolysed tissue, follicular cyst, simple mucinous cyst and was without evidence of malignancy.

Among malignant cases, most common diagnosis was germ cell tumour, granulosa cell tumour and serous cyst adenocarcinoma ( $n=4 ; 12.9 \%$ each). There were $2(6.5 \%)$ cases reported as mucinous cyst adenocarcinoma and malignant ovarian tumour respectively.

\begin{tabular}{|c|c|c|c|}
\hline \multirow{2}{*}{$\begin{array}{l}\text { Frozen Section } \\
\text { Diagnosis }\end{array}$} & \multicolumn{2}{|c|}{ Final Diagnosis (HPE) } & \multirow{2}{*}{ Total } \\
\hline & Malignant & Benign & \\
\hline Malignant (Fig. 1, 3, 4) & 12 & 0 & 12 \\
\hline Benign (Fig. 2) & 2 & 14 & 16 \\
\hline Total & 14 & 14 & 28 \\
\hline \multicolumn{4}{|c|}{$\begin{array}{c}\text { Table 7. Diagnostic Efficacy of Frozen Section Diagnosis } \\
\text { against Final Diagnosis for Malignancy }(n=28)\end{array}$} \\
\hline
\end{tabular}

Sensitivity $=85.7 \%$; Specificity $=100 \%$; PPV= 100\%; NPV= 87.5\%; Accuracy=92.9\%.

Frozen sections could be analysed in 28 cases only. On comparing the diagnosis of 28 cases of frozen section diagnosis, all the 12 cases diagnosed as malignant by frozen section were found to be malignant on final diagnosis too, whereas of the 16 cases diagnosed as benign 14 were diagnosed as benign and 2 diagnosed as malignant on final diagnosis. Thus, the technique had 12 true positive, no false positive, two false negative and 14 true negative cases, thereby showing a sensitivity, specificity, positive predictive value, negative predictive value and accuracy values of $85.7 \%, 100 \%, 100 \%, 87.5 \%$ and $92.9 \%$ respectively.

\begin{tabular}{|c|c|c|c|}
\hline \multirow{2}{*}{ Aspirate Diagnosis } & \multicolumn{2}{|c|}{ Final Diagnosis } & \multirow{2}{*}{ Total } \\
\cline { 2 - 3 } & Malignant & Benign & \\
\hline Malignant (Fig. 1, 3, 4) & 6 & 0 & 6 \\
\hline Benign (Fig. 2) & 9 & 15 & 24 \\
\hline Total & $\mathbf{1 5}$ & $\mathbf{1 5}$ & $\mathbf{3 0}$ \\
\hline Table 8. Diagnostic Efficacy of Aspirate Diagnosis \\
against Final Diagnosis for Malignancy (n = 30)
\end{tabular}

Sensitivity $=40 \%$; Specificity $=100 \%$; PPV $=100 \%$; NPV= $62.5 \%$; Accuracy $=70 \%$.

A total of 30 cases could be analysed using aspirate diagnosis. On comparing the diagnosis of these 30 cases against final diagnosis, it was seen that all the 6 malignant cases diagnosed by aspiration were malignant on final diagnosis too. However, of the remaining 24 cases diagnosed as benign by aspiration, 15 were benign and 9 were malignant. Thus, a total of 6 cases were true positive, none was false positive, 9 were false negative and 15 were true negative. Correspondingly, aspiration had a sensitivity of $40 \%$, specificity of $100 \%$, positive predictive value of $100 \%$, negative predictive value of $62.5 \%$ and accuracy of $70 \%$.

\begin{tabular}{|c|c|c|c|}
\hline \multirow{2}{*}{ Imprint Diagnosis } & \multicolumn{2}{|c|}{ Final Diagnosis } & \multirow{2}{*}{ Total } \\
\cline { 2 - 3 } & Malignant & Benign & \\
\hline Malignant (Fig. 1, 3, 4) & 5 & 0 & 5 \\
\hline Benign (Fig. 2) & 10 & 15 & 25 \\
\hline Total & $\mathbf{1 5}$ & $\mathbf{1 5}$ & $\mathbf{3 0}$ \\
\hline $\begin{array}{r}\text { Table 9. Diagnostic Efficacy of Imprint Diagnosis } \\
\text { against Final Diagnosis for Malignancy (n = 30) }\end{array}$ \\
\hline
\end{tabular}

Sensitivity $=33.3 \%$; Specificity $=100 \%$; PPV= 100\%; NPV= $60 \%$; Accuracy $=66.7 \%$.

A total of 30 cases could be analysed using imprint diagnosis. All the 5 malignant cases diagnosed by imprint cytology were malignant on final diagnosis too. However, of the remaining 25 cases diagnosed as benign by imprint, 15 were benign and 10 were malignant. Thus, a total of 5 cases were true positive, none was false positive, 10 were false negative and 15 were true negative. Correspondingly, imprint had a sensitivity of $33.3 \%$, specificity of $100 \%$, positive predictive value of $100 \%$, negative predictive value of $60 \%$ and accuracy of $66.7 \%$.

\begin{tabular}{|c|c|c|c|c|}
\hline Variable & Frozen Section & $\begin{array}{l}\text { Aspirate } \\
\text { Cytology }\end{array}$ & $\begin{array}{c}\text { Imprint } \\
\text { Cytology }\end{array}$ & Final Diagnosis \\
\hline Total cases tested & 28 & 30 & 30 & 31 \\
\hline Malignant & 12 & 6 & 5 & 16 \\
\hline \% Malignancy rate & 42.9 & 20 & 16.7 & 51.6 \\
\hline
\end{tabular}




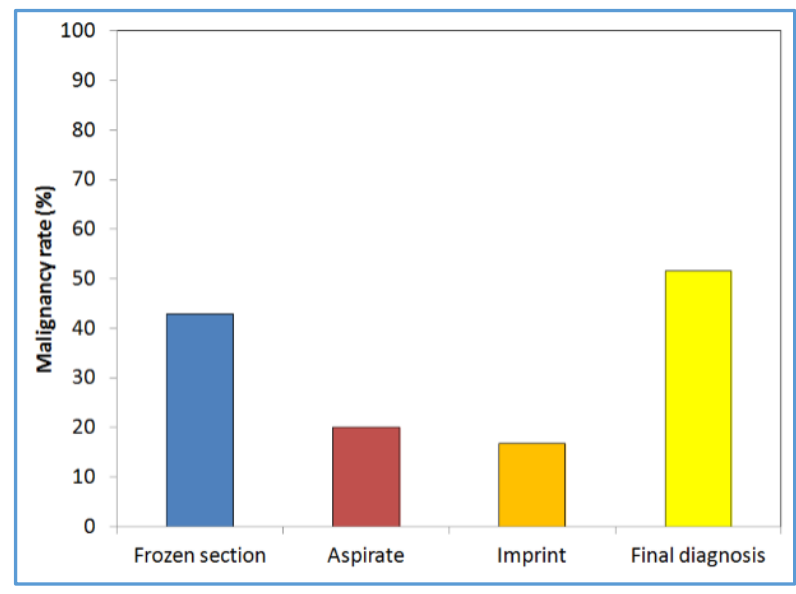

Graph 5
Malignancy rate was maximum in final diagnosis (51.6\%) followed by frozen section (42.9\%), aspirate cytology (20\%) and imprint cytology (16.7\%). Statistically, no significant difference in malignancy rate by frozen section and final diagnosis was observed $(p=0.739)$. Final HPE diagnosis had significantly higher malignancy rates as compared to both aspirate cytology and imprint cytology ( $\mathrm{p}<0.05)$.

Frozen section had higher malignancy rates as compared to both aspirate and imprint techniques, but difference was significant only for comparison between frozen section and imprint techniques $(\mathrm{p}=0.029)$.

\section{DISCUSSION}

Frozen Section Techniques' Statistical Parameters

85.7\% sensitivity, 100\% specificity, 100\% Positive Predictive Value (PPV), 87.5\% Negative Predictive Value (NPV) and 92.9\% accuracy in regard to frozen section technique. Other studies done in the past regarding the use of frozen section in intraoperative diagnoses of ovarian tumours are listed below.

\begin{tabular}{|c|c|c|c|c|c|c|c|}
\hline Author & $\begin{array}{c}\text { Yea } \\
\mathbf{r}\end{array}$ & $\begin{array}{c}\text { Sample } \\
\text { Size }\end{array}$ & Sensitivity & Specificity & PPV & NPV & Accuracy \\
\hline $\begin{array}{c}\text { Stewart } \\
\text { CJ et al[3] }\end{array}$ & $\begin{array}{c}201 \\
0\end{array}$ & 402 cases & - & - & - & - & $97.8 \%$ \\
\hline $\begin{array}{l}\text { U. D. } \\
\text { Bafna } \\
\text { et al[9] }\end{array}$ & $\begin{array}{c}201 \\
3\end{array}$ & 135 cases & $\begin{array}{c}\text { Benign- } 90.4 \% \\
\text { Borderline- } \\
31.2 \% \\
\text { Malignant- } 91.5 \%\end{array}$ & $\begin{array}{c}\text { Benign- } 82.6 \% \\
\text { Borderline- } 94 \% \\
\text { Malignant- } \\
98.2 \%\end{array}$ & - & - & $\begin{array}{c}\text { 89.5\%- Serous } \\
48.2 \%- \\
\text { Mucinous } \\
100 \%-\text { Germ } \\
\text { cell tumour } \\
85 \% \text { - Sex cord }\end{array}$ \\
\hline $\begin{array}{c}\text { Virach } \\
\text { Wootipoo } \\
\text { m et al[16] }\end{array}$ & $\begin{array}{c}200 \\
6\end{array}$ & 229 cases & $\begin{array}{c}\text { Benign- } 98.2 \% \\
\text { Borderline- } \\
\text { 57.1\% malignant- } \\
\quad 86.1 \%\end{array}$ & $\begin{array}{c}\text { Benign- 87.0\% } \\
\text { Borderline- } \\
96.4 \% \\
\text { malignant- } \\
98.5 \%\end{array}$ & $\begin{array}{c}\text { Benign- 89.5\% } \\
\text { Borderline- } \\
63.2 \% \\
\text { Malignant- } \\
97.1 \%\end{array}$ & $\begin{array}{c}\text { Benign- 97.8\% } \\
\text { Borderline- } \\
95.4 \% \\
\text { Malignant- } \\
92.3 \%\end{array}$ & $89.7 \%$ \\
\hline $\begin{array}{l}\text { Amita } \\
\text { Maheshw } \\
\text { ari et } \\
\text { al[17] }\end{array}$ & $\begin{array}{c}200 \\
6\end{array}$ & 241 cases & $\begin{array}{c}\text { Benign- } 100 \% \\
\text { Borderline- } \\
45.5 \% \\
\text { Malignant- } 93.5 \%\end{array}$ & $\begin{array}{c}\text { Benign- 93.2\% } \\
\text { Borderline- } \\
98.5 \% \\
\text { malignant- } \\
98.3 \%\end{array}$ & & & $91.2 \%$ \\
\hline $\begin{array}{c}\text { Arikan } \\
\text { Ilker et } \\
\text { al[18] }\end{array}$ & $\begin{array}{c}201 \\
1\end{array}$ & 266 cases & $\begin{array}{c}\text { Benign- 100\% } \\
\text { Borderline- } \\
77.8 \% \\
\text { Malignant- } 71.4 \%\end{array}$ & $\begin{array}{c}\text { Benign- 83.8\% } \\
\text { Borderline- } \\
98.4 \% \\
\text { Malignant- } \\
100 \%\end{array}$ & - & - & - \\
\hline $\begin{array}{l}\text { Sennur } \\
\text { Ilvan } \\
\text { et al[19] }\end{array}$ & $\begin{array}{c}200 \\
5\end{array}$ & 617 cases & $\begin{array}{c}\text { Benign- } 100 \% \\
\text { Borderline- } 87 \% \\
\text { Malignant- } 87 \%\end{array}$ & $\begin{array}{c}\text { Benign- } 97 \% \\
\text { Borderline- } 98 \% \\
\text { Malignant- } \\
100 \%\end{array}$ & - & - & $97 \%$ \\
\hline $\begin{array}{l}\text { Atif Ali } \\
\text { Hashmi } \\
\text { et al[7] }\end{array}$ & $\begin{array}{c}201 \\
6\end{array}$ & 141 cases & $\begin{array}{c}\text { Benign- } 100 \% \\
\text { Borderline- } 83 \% \\
\text { Malignant- } 96 \%\end{array}$ & $\begin{array}{c}\text { Benign- 97\% } \\
\text { Borderline- 99\% } \\
\text { Malignant- } \\
100 \%\end{array}$ & - & - & $99.00 \%$ \\
\hline Our Study & $\begin{array}{c}201 \\
6\end{array}$ & 28 cases & $85.7 \%$ & $100 \%$ & $100 \%$ & $87.5 \%$ & $92.9 \%$ \\
\hline \multicolumn{8}{|c|}{ Table 11. Comparative Data of Different Studies Performed regarding Frozen Section Technique } \\
\hline
\end{tabular}


Thus, our study results show higher sensitivity, specificity, positive predictive value and negative predictive value in regard to frozen section technique.

Some of the researchers have suggested that one frozen section should be performed for every $10 \mathrm{~cm}$ diameter of tumour for very large mucinous ovarian tumours to improve accuracy. ${ }^{[20]}$ They suggested the value of frozen section of ovarian tumours also lies in establishing whether an ovarian tumour is primary or metastatic.[7]

Intraoperative Cytology Techniques' Statistical Parameters

\begin{tabular}{|c|c|c|c|c|c|c|c|}
\hline Author & Year & Cases & Sensitivity & Specificity & PPV & NPV & Overall Accuracy \\
\hline Stewart CJ et al[3] & 2010 & 402 cases & - & - & - & - & - \\
\hline Khunamornpong S et al[6] & 2012 & 50 cases & $95.8 \%$ & $96 \%$ & - & - & $95.8 \%$ \\
\hline Nishat Afroz et al[14] & 2009 & 120 cases & - & - & - & - & - \\
\hline Chandrashekhar T et al[15] & 2014 & 30 cases & - & - & - & - & - \\
\hline Renu Jain et al[21] & 2015 & 68 cases & $97.7 \%$ & $91.37 \%$ & $95.56 \%$ & $95.65 \%$ & $95.6 \%$ \\
\hline
\end{tabular}

1. Cystadenocarcinoma- Cytology, 40x H and E Histology, Frozen, Fresh Gross (Clockwise)

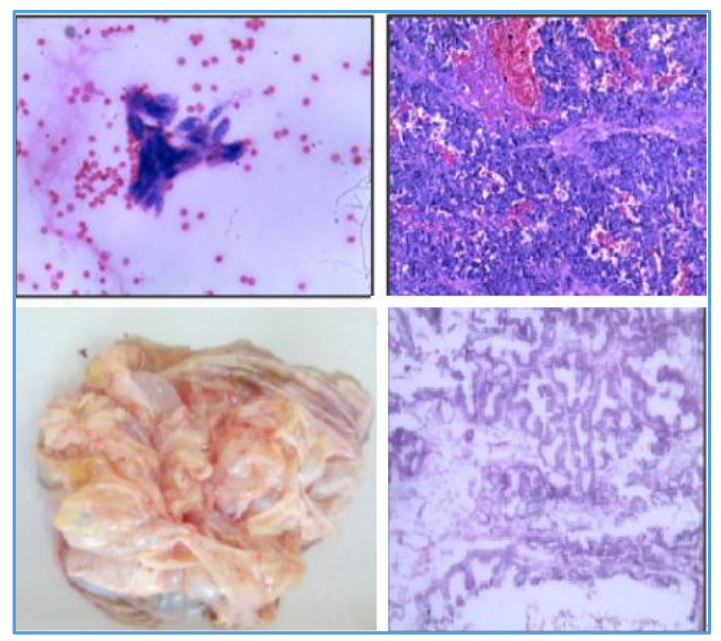

2. Endometriotic and Simple Cysts- Cytology did not reveal adequate Smear.

Gross and Frozen (Right corresponding 40x H and E).

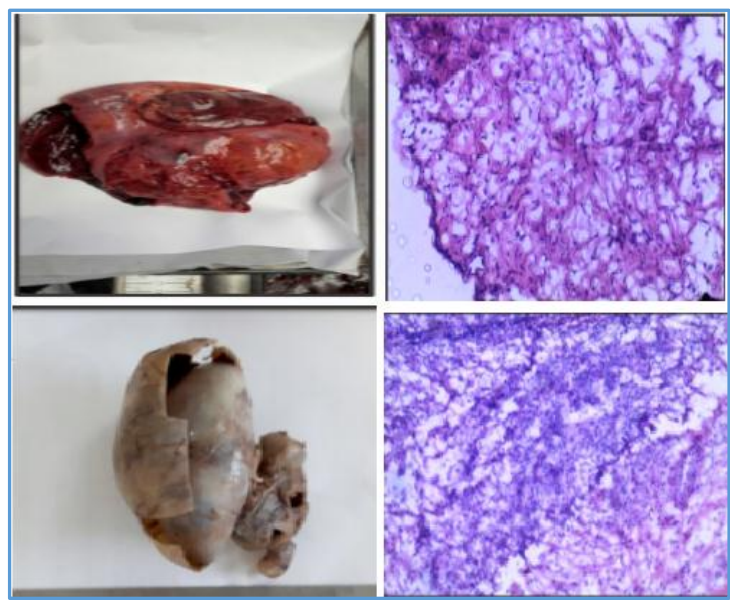

3. Malignant Mixed Germ Cell Tumour- Frozen could not be done.

40X Giemsa Cytology, Gross, H and E 40x Histology (Clockwise).

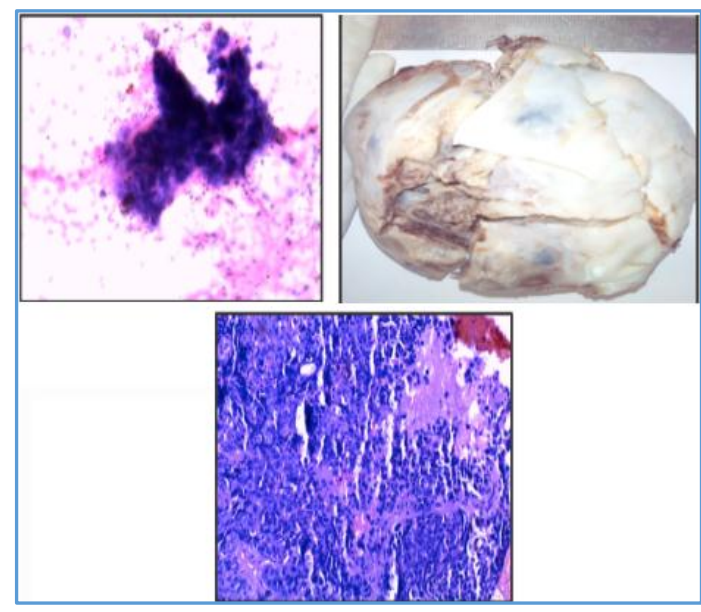

4. Granulosa Cell Tumour- Cytology did not reveal adequate Smear.

40x H and E Histology, Gross, Frozen (Clockwise).

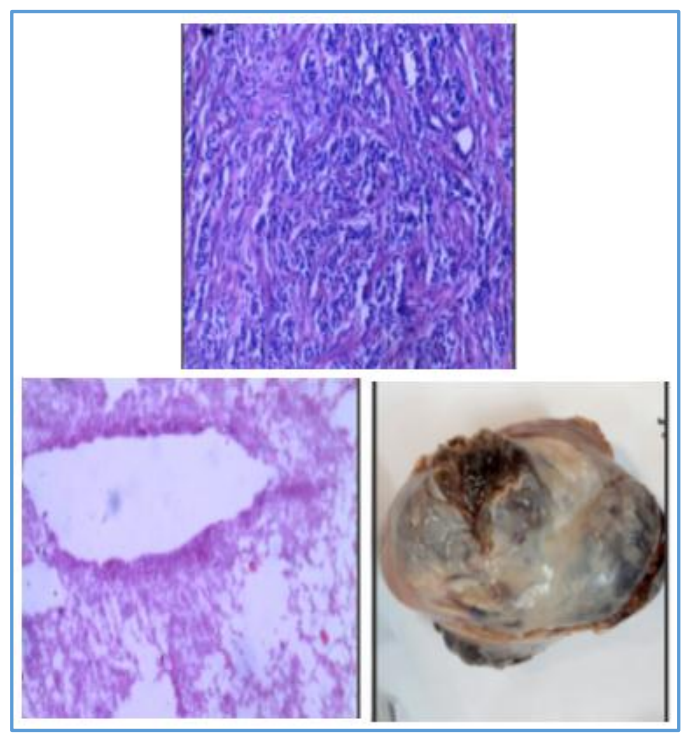




\section{Limitations of Study}

1. Power failure resulted in reduction of number of samples initially planned to be taken up for comparison of the three arms.

2. All cases of ovarian diseases (enrolled in KGMU Obstetrics Department) could not be included because of referral to surgical/medical oncology departments of same or other institutes for further management.

3. Interpretation of error in benign or borderline cases in frozen samples.

4. Morphology interpretation error.

5. In some cases, cytology could not be done in our study due to very small amount of ovarian tissue provided.

\section{CONCLUSION}

1. Frozen section is a technique that determines intraoperatively whether an ovarian mass is benign or malignant and helps determine the extent of surgery.

2. Frozen section has a higher sensitivity, specificity, positive predictive value, negative predictive value and accuracy than aspirate and imprint cytology.

3. Aspirate cytology has a higher sensitivity, specificity, positive predictive value, negative predictive value and accuracy than imprint cytology.

4. Gold standard that detects whether the ovarian lesion is malignant or benign is histopathological examination and immunohistochemistry wherever needed.

\section{REFERENCES}

[1] Baker P, Oliva E. A practical approach to intraoperative consultation in gynaecological pathology. International Journal of Gynaecological Pathology 2008;27(3):353-65.

[2] Di Saia PJ, Creasman WT. The adnexal mass and early ovarian cancer. Chapter $10.7^{\text {th }}$ edn. Clinical Gynecologic Oncology: Mosby 2008:283-312.

[3] Stewart CJ, Brennan BA, Hammond IG, et al. Intraoperative assessment of ovarian tumors: a 5-year review with assessment of discrepant diagnostic cases. International Journal of Gynecological Pathology 2006;25(3):216-22.

[4] Abdelghany AM, Arafa EM, Madkour NM, et al. Comparative study between intraoperative frozen section and scrape smear cytology in the diagnosis of ovarian neoplasm. Open J Obstet Gynecol 2015;5(1):28-35.

[5] Kolte SS, Satarkar RN. Role of scrape cytology in the intraoperative diagnosis of tumor. Journal of Cytology 2010;27(3):86-90.

[6] Khunamornpong, S. and Siriaunkgul S. Scrape cytology of the ovaries: potential role in intraoperative consultation of ovarian lesions. Diagnostic Cytopathology 2003;28(5):250-7.

[7] Hashmi AA, Naz S, Edhi MM, et al. Accuracy of intraoperative frozen section for the evaluation of ovarian neoplasms. World Journal of Surgical Oncology 2016;14:91.
[8] Santín AC, Sica A, Melesi S, et al. Contribution of intraoperative cytology to the diagnosis of ovarian lesions. Acta Cytologica 2011;55(1):85-91.

[9] Subbian A, Devi UK, Bafna UD. Accuracy rate of frozen section studies in ovarian cancers: a regional cancer institute experience. Indian Journal of Cancer 2013;50(4):302-5.

[10] Scopa CD, Melachrinou M, Apessou D, et al. Tissue imprints in surgical pathology: a rapid intraoperative diagnostic aid. Diagn Cytopathol 1990;6(1):5-8.

[11] Shidham VB, Dravid NV, Grover S, et al. Role of scrape cytology in rapid intraoperative diagnosis. Value and limitations. Acta Cytol 1984;28(4):477-82.

[12] Souka S, Kamel M, Rocca M. et al. The combined use of cytological imprint and frozen section in the intraoperative diagnosis of ovarian tumors. International Journal of Gynecology and Obstetrics 1990;31(1):43-6.

[13] Helpap B, Tschubel K. Comparison between frozen section histology and touch cytology of the breast. Fortschr Med 1977;95(25):2119-20.

[14] Khan N, Afroz N, Aqil B, et al. Neoplastic and nonneoplastic ovarian masses: diagnosis on cytology. Journal of Cytology 2009;26(4):129-33.

[15] Chandrashekhar T, Govindraja E, Chandana N. et al. The diagnostic accuracy of intra-operative imprint cytology in ovarian tumors. Jounal of Evolution of Medical And Dental Sciences 2014;3(43):10614-25.

[16] Wootipoom V, Dechsukhum C, Hanprasertpong J, et al. Accuracy of intra-operative frozen section in diagnosis of ovarian tumors. Journal of the Medical Association of Thailand 2006;89(5):577-82.

[17] Maheshwari A, Gupta S, Kane S, et al. Accuracy of intraoperative frozen section in the diagnosis of ovarian neoplasms: experience at a tertiary oncology center. World Journal of Surgical Oncology 2006;4:12.

[18] Ilker A, Brut A, Muge $\mathrm{H}$, et al. Accuracy of intraoperative frozen section in the diagnosis of ovarian tumours. Journal of Pakistan Medical Association. 2011;61:856.

[19] Ilvan S, Ramazanoglu R, Akyildiz EU, et al. The accuracy of frozen section (intraoperative consultation) in the diagnosis of ovarian masses. Gynaecologic Oncology 2005;97(2): 395-9.

[20] Vijaykumar A. The diagnostic utility of intraoperative cytology in the management of ovarian tumours. Journal of Clinical And Diagnostic Research 2013;7(6):1047-50.

[21] Jain R, Jain V, Dutta S, et al. Role of Intra-operative cytology in the diagnosis of ovarian neoplasms. International Journal of Scientific Study 2015;3(5): 72-5. 